\title{
Peritoneal Dialysis in Renal Replacement Therapy for Patients with Acute Kidney Injury
}

\author{
Naheed Ansari \\ Division of Nephrology, Department of Medicine, Jacobi Medical Center, 1400 Pelham Parkway, South Bronx, NY 10461, USA \\ Correspondence should be addressed to Naheed Ansari, naheed.ansari@nbhn.net \\ Received 16 August 2010; Revised 8 February 2011; Accepted 21 March 2011 \\ Academic Editor: Achim Jörres \\ Copyright () 2011 Naheed Ansari. This is an open access article distributed under the Creative Commons Attribution License, \\ which permits unrestricted use, distribution, and reproduction in any medium, provided the original work is properly cited. \\ Peritoneal dialysis (PD) was the first modality used for renal replacement therapy (RRT) of patients with acute kidney injury (AKI) \\ because of its inherent advantages as compared to Hemodialysis. It provides the nephrologist with nonvascular alternative for renal \\ replacement therapy. It is an inexpensive modality in developing countries and does not require highly trained staff or a complex \\ apparatus. Systemic anticoagulation is not needed, and it can be easily initiated. It can be used as continuous or intermittent \\ procedure and, due to slow fluid and solute removal, helps maintain hemodynamic stability especially in patients admitted to \\ the intensive care unit. PD has been successfully used in AKI involving patients with hemodynamic instability, those at risk of \\ bleeding, and infants and children with AKI or circulatory failure. Newer continuous renal replacement therapies (CRRTs) are \\ being increasingly used in renal replacement therapy of AKI with less use of PD. Results of studies comparing newer modalities of \\ CRRT versus acute peritoneal dialysis have been conflicting. PD is the modality of choice in renal replacement therapy in pediatric \\ patients and in patients with AKI in developing countries.
}

\section{Introduction}

Acute kidney injury occurs in hospitals and is seen in up to $5 \%$ of hospitalized patients. $0.5 \%$ of the patients with AKI require dialysis [1]. Acute kidney injury occurs more frequently in intensive care unit (ICU) as part of the multiorgan failure and is usually associated with higher mortality rate and increased dialysis requirement in ICU setting.

There are two major types of dialysis available these days: Peritoneal dialysis (PD) and Hemodialysis (HD). PD was the modality first used for treatment of AKI [2]. Intermittent PD was widely used in the 1970s due to its various advantages. Later, continuous PD therapies became available through automated cycling machines (cyclers).

In western countries, peritoneal dialysis is not commonly used in dialytic management of acute kidney injury due to the availability of newer HD techniques and development of continuous renal replacement therapies (CRRTs). The new technological advances in the extracorporeal circuit of HD successfully compete with traditional advantages of PD. The limiting factors are the slow efficiency of the procedure, limited value in patients with recent intra-abdominal surgery or intra-abdominal pathology, and rise in intra-abdominal pressure which may compromise pulmonary function. The rise in intra-abdominal pressure may have deleterious effects in patients with acute lung injury or the acute respiratory distress syndrome (ARDS). High glucose content of the PD solution can evoke hyperglycemia and other metabolic derangements. Increased carbon dioxide production is associated with an increase in respiratory quotient to $>1.0$ which may exacerbate respiratory failure in patients with compromised lung function.

This paper paper covers the use of peritoneal dialysis in the setting of AKI especially regarding indications, various techniques, prescription of acute $\mathrm{PD}$, and various complications encountered during the procedure.

\section{Indications of Acute PD}

The indications for acute PD can be divided into two groups: renal and nonrenal.

2.1. Renal Indications. Peritoneal dialysis is an advantageous modality for RRT in AKI (Table 1). It is indicated in both 
TABLE 1: Renal indications of peritoneal dialysis in AKI.

(1) RRT in the treatment of AKI in children

(2) Hemodynamically unstable patients

(3) The presence of bleeding diasthesis or hemorragic conditions contraindicating placement of vascular access for hemodialysis or anticoagulation

(4) Patients with difficult vascular access placement

(5) Removal of high molecular weight toxins (10 kD)

TABLE 2: Nonrenal indications.

(1) Acute pancreatitis

(2) Clinically significant hypothermia or hyperthermia

(3) Refractory heart failure

(4) Liver failure

(5) Infusion of drugs and nutrients as a supportive therapy in critically ill patients

renal and nonrenal-related conditions where it is indicated due to advantages of the PD modality. PD can easily meet treatment goals for AKI patients, maintaining adequate fluid, electrolyte, and acid base balances. It also allows the use of other supportive measures like use of intravenous infusion of sedatives, vasopressors in hemodynamically unstable patients, and total parenteral nutrition to continue without limitation until the recovery of renal function. However, as compared to HD, PD is less effective in severe acute illnesses like pulmonary edema, poisoning, or drug overdose, and hypercatabolic states.

Usually small molecular clearance is lower with PD than that achieved with conventional HD. This is because small molecule concentration in peritoneal dialysate is approximately $30 \%-50 \%$ of the equivalent serum values after 1 hour of PD and 50\%-80\% after a 4 -hour dwell [3]. On the other hand, the clearance of higher molecular weight solutes is higher with continuous PD than with HD [4].

The ultrafiltration rate is maximal at the beginning of a $\mathrm{PD}$ exchange when glucose concentration is at its maximum. As the glucose is absorbed and its concentration further drops due to movement of ultrafiltrate into the peritoneal cavity, there is further fall in the ultrafiltration rate. As a result of which the intraperitoneal volume peaks at about 120-180 minutes of dwell.

Acute PD is the preferred mode of RRT in children [5]. In patients with hemodynamic instability due to various reasons, $\mathrm{PD}$ is preferable to conventional $\mathrm{HD}$ [6]. PD can meet treatment targets for AKI and can maintain adequate fluid, electrolyte, solute clearances, and acid base balance in patients with AKI.

Earlier studies have shown that patients treated with PD had lower mortality rates and a higher incidence of renal recovery than did similar patients treated with $\operatorname{HD}[7,8]$.

2.2. Nonrenal Indications for Acute PD. PD can be used in various extra renal conditions (Table 2). In acute hemorrhagic pancreatitis, PD helps in the removal of bioactive
TABle 3: Peritoneal dialysis is contraindicated in the following clinical Situations.

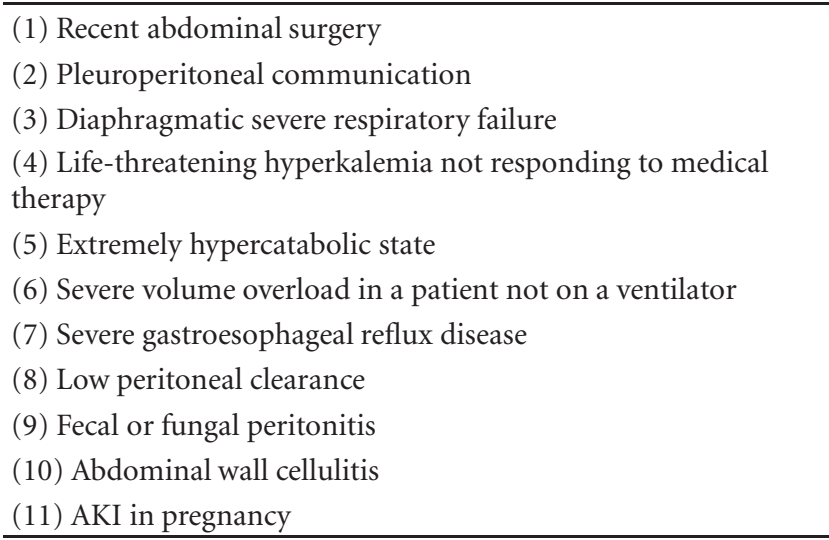

substances presumed to be responsible for systemic inflammation associated with acute pancreatitis [9-11]. However, a multicenter prospective study found no difference in the mortality or complication rate for patients who received standard supportive therapy with or without hourly 2-L peritoneal dialysis exchanges for 3 days [12].

Clinically significant hypothermia or hyperthermia can be managed with PD where heated or cold peritoneal solutions can be used to maintain core temperature. This is usually done in patients with either hypothermia or hyperthermia who do not respond to conventional therapy [13-15].

Congestive Heart Failure (CHF) refractory to medical therapy can be treated with peritoneal dialysis when there is inadequate response to diuretics in severely volume overloaded state [16-19].

In patients with fulminant liver failure, $\mathrm{PD}$ has been used because it avoids need for anticoagulation. It corrects fluid and electrolyte disorder and may reduce the risk of hypoglycemia and hypothermia as compared to charcoal hemoperfusion [9]. PD may help in the removal of toxins like ammonia, bilirubin, and free fatty acids.

PD may be used as route for delivery of nutrients like glucose and amino acids and certain drugs in severely ill patients admitted to intensive care unit. This route alone may not be enough in severely malnourished individuals [20-22].

\section{Contraindications to Acute PD}

There are several relative contraindications to acute PD (Table 3), such as recent abdominal surgery, peritonitis (fecal or fungal), and known pleuroperitoneal fistula. Presence of abdominal drain increases the risk of local infection. The presence of abdominal hernia or intra-abdominal adhesions makes PD difficult.

The use of PD is relatively contraindicated in the presence of the abdominal wall cellulitis which may progress to peritonitis. It is also contraindicated in severe gastroesophageal reflux disease, and in adynamic ileus which may decrease efficiency of peritoneal dialysis. In patients with relative respiratory insufficiency, the use of intraperitoneal fluid may 


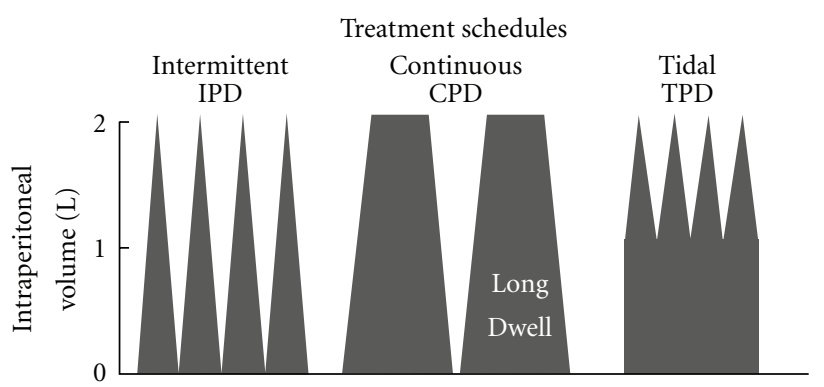

FIGURE 1: Basic mechanisms operating in various peritoneal dialysis regimens.

increase intra-abdominal pressure and hence compromise lung function and respiratory gas exchange. Acute PD is ineffective in treatment of life threatening hyperkalemia [23]. Use of PD in life threatening Hyperkalemia should be reserved in situations when HD is not available. It is not the best modality of RRT in hypercatabolic patients with a high load of azotemia [24].

\section{Regimens of Acute PD}

PD can be performed intermittently or continuously and either manually or via an automated device [25]. Performance of acute PD requires only an intact peritoneal cavity [25]. Various regimens of acute PD are shown in Figure 1.

\subsection{IPD (Classical Intermittent Peritoneal Dialysis). IPD is} the most commonly used regimen of PD. The usual exchange time is 1 hour. It can be done either manually or by using a cycling device programmed to deliver a predetermined volume of peritoneal dialysis fluid and to drain the peritoneal cavity at fixed intervals. Short exchanges of 1-2 L performed in sessions of 16-24 hours twice or three times weekly can deliver doses of about 40-60 L per session (80-180 L/week). This type of PD has been extensively used in $\operatorname{ARF}[24,26]$.

4.2. CPD (Continuous Peritoneal Dialysis). It is a modified form of CAPD in which manual exchange is done every 3-6 hours depending upon patient clearance and fluid removal requirements. It provides PD in an inpatient setting [27]. It is a simple procedure, has low cost, and is less labor intensive. It differs from IPD because it uses relatively long dwell times and uses multiple daily exchanges, in which peritoneal dialysis fluid is instilled and drained continuously every 3 6 hours. These exchanges can be performed manually or by a cycler. It maintains a low flow continuous system, which itself maintains stable blood levels of nitrogenous products. It is commonly done in developing countries because of the low cost and is less labor intensive. The disadvantage is that clearances may not be adequate especially in hypercatabolic patients because of a lower dialysate flow rate.

4.3. TPD (Tidal Peritoneal Dialysis). With this technique, after an initial exchange of the peritoneal cavity with peritoneal dialysis fluid, only a portion of dialysate is drained.
The drained volume is replaced by fresh dialysate with each cycle leaving a variable amount of dialysate in constant contact with the peritoneal membrane until the end of dialysis session when the fluid is drained as completely as possible. Tidal PD was originally designed to optimize solute clearances [28] by leaving constant tidal volume of dialysate in the peritoneal cavity throughout the dialysis session. Clinical studies have not confirmed increased solute clearance with use of TPD [29-31]. The tidal volume is usually one half of initial filling by the cycler of a large (2L) volume of solution during a dialysis session that lasts for $8-10$ hours.

\section{Peritoneal Access}

Access is one of the important determinants of successful peritoneal dialysis. A peritoneal catheter is inserted in the peritoneal cavity to gain access to the peritoneal space for initiation of dialysis.

There are two different types of peritoneal catheters.

(1) Semirigid Acute Catheter. This can be inserted at bedside by a nephrologist and does not need surgical help [32]. It is usually inserted under local anesthesia and hence avoids complications of general anesthesia. The major disadvantages are high risk of infection, discomfort in an awake patient, and risk of bowel perforation. This type of catheter cannot be left in place for more than 72 hours due to high risk of peritonitis $[32,33]$.

(2) Cuffed Permanent Catheter. This is usually a Tenckhoff catheter. It has a much lower risk of infection, can be used immediately after insertion, has a lower risk of bowel perforation, and avoids the need for repeated punctures in intra-abdominal cavity as with semirigid catheters. This catheter is preferred in patients with acute PD on a cycler because this catheter does not trigger alarms on CCPD as sometimes experienced with use of semirigid catheters.

This type of catheter is placed under local anesthesia and requires surgical expertise for insertion. The hemodynamic status of the patient may limit the feasibility of this catheter especially in sick ICU patients.

\section{Acute Peritoneal Dialysis Prescription}

After the insertion of an acute or chronic peritoneal catheter preferably chronic if possible, $\mathrm{PD}$ orders need to be individualized depending upon hemodynamic status of the patient, laboratory work, and volume status. A standardized form with complete and clear specifications for procedure should be used if available (Table 7). PD orders must be reviewed and written daily as patients with AKI can fluctuate their acid base and electrolyte milieu. The components of PD orders are multiple and involve the following (Table 4).

A usual dialysis session lasts for 48-72 hours and each exchange is done over one hour. A typical session of acute peritoneal dialysis has $48-72$ exchanges. However, the length of a PD session can vary depending on the cause and 
TABLE 4: Components of acute PD prescription.

(1) Length of the dialysis session

(2) Dialysate composition

(3) Exchange volume

(4) Inflow and outflow periods

(5) Dwell time

(6) Number of exchanges

(7) Additives

(8) Monitoring of fluid balance

TABle 5: Composition of peritoneal dialysis fluid.

(1) Sodium 132-134 ( $\mathrm{mmol} / \mathrm{L})$

(2) Potassium 0-2 ( $\mathrm{mmol} / \mathrm{L})$

(3) Calcium 1.25-1.75 ( $\mathrm{mmol} / \mathrm{L})$

(4) Magnesium 0.25-0.75 ( $\mathrm{mmol} / \mathrm{L})$

(5) Chloride 95-106 ( $\mathrm{mmol} / \mathrm{L})$

(6) Lactate $35-40(\mathrm{mmol} / \mathrm{L})$ or $\mathrm{HCO} 3(34 \mathrm{mmol} / \mathrm{L})$

(7) Glucose $1.5-4.25(\mathrm{~g} / \mathrm{dL})$

(8) $\mathrm{pH}$ (Neutral and physiological in newer peritoneal dialysis fluid preparations)

duration of AKI, need for water and solute removal, and the risk of infection.

PD fluid (PDF) is available in standard monohydrate glucose concentrations of $1.5,2.5$, and $4.25 \mathrm{~g} / \mathrm{dL}$ and various electrolyte concentrations (Table 5). PD fluid should be warmed to body temperature prior to infusion to avoid discomfort and enhance solute transport.

To obtain better ultrafiltration, it is reasonable to initiate acute PD in most patients with the $2.5 \mathrm{~g} / \mathrm{dL}$ PD fluid. An initial glucose concentration of $1.5 \mathrm{~g} / \mathrm{dL}$ may be more appropriate in patients with only moderate amounts of fluid overload and in those who are hemodynamically unstable.

PD fluid with a higher glucose concentration can be substituted based on the amount of fluid removed and the patient's hemodynamic parameters. With a standard regimen, such as a two-liter exchange volume and one-hour dwell time, the following average amounts of fluid can be removed over a 24-hour period:

(i) 2.5 liters with $1.5 \mathrm{~g} / \mathrm{dL}$ glucose,

(ii) 4.5 liters with $2.5 \mathrm{~g} / \mathrm{dL}$ glucose,

(iii) 8.5 liters with $4.25 \mathrm{~g} / \mathrm{dL}$ glucose.

Various types of glucose concentration are available to use in acute PD prescription (Table 6).

(i) $1.5 \mathrm{~g} / \mathrm{dL}$ PD fluid contains 27.2 grams of glucose in 2 liter bag. It usually gives ultrafiltration of $50-150 \mathrm{~mL}$ per hour when using a 2-L exchange volume over 60 minute exchange time. It is the most commonly used fluid in acute PD.

(ii) $2.5 \mathrm{~g} / \mathrm{dL}$ PD fluid can give UF of $100-300 \mathrm{cc}$ per exchange volume of $2 \mathrm{~L}$ and exchange time of one hour. $2 \mathrm{~L}$ bag of $2.5 \mathrm{~g} / \mathrm{dL}$ of PDF contains 45.4 grams of glucose.

(iii) $4.25 \mathrm{~g} / \mathrm{dL}$ PD fluid contains 77.2 grams of glucose in 2 liter bag and can give rise to UF of 300$400 \mathrm{cc} /$ exchange. This hypertonic fluid is usually used in patients with volume overload like CHF. Continued use of the $4.25 \mathrm{~g} / \mathrm{dL}$ fluid could result in removal of $7.2-9.6 \mathrm{~L}$ per day which can be very dangerous since it can induce hemodynamic instability due to massive ultrafiltration, and high glucose content of hypertonic PDF is deleterious to the peritoneal membrane. This degree of UF is not required usually and one can use combination of glucose concentrations to attain level of UF desired.

The most practical way to achieve fluid removal is by mixing and matching low and high glucose concentration adequate fluid. Once the patient is euvolemic, the dialysis fluid should be switched to a glucose concentration of $1.5 \mathrm{~g} / \mathrm{dL}$ and the rate of exchange should be slowed.

Exchange volume is the amount of PD fluid instilled into the peritoneal cavity during an exchange. The volume instilled depends on the intraperitoneal pressure (IPP), the presence of pulmonary disease or mechanical ventilation, and the presence of abdominal hernia. An average sized adult can tolerate 2-L exchanges but in smaller patients, those with pulmonary disease or those with abdominal or inguinal hernias, the exchange volume should be reduced. In pediatric patients with AKI requiring dialysis, exchange volume is based on body weight. Usually volume of $30 \mathrm{~mL} / \mathrm{Kg}$ body weight is used for PD.

The intraperitoneal pressure rises linearly with higher volume of intraperitoneal fluid used. Intraperitoneal pressure is higher in patients with higher body mass index. Age, gender, weight, height, body surface area (BSA), and Diabetes Mellitus, do not correlate with IPP [34]. The peritoneal dialysis fluid volume should be reduced in patients with a pulmonary disease (like pneumonia or COPD) or respiratory failure. The rise in intra-abdominal pressure due to PD fluid may hamper diaphragmatic excursions needed for respirations. Similarly, patients with abdominal wall or inguinal hernia may require less volume to prevent a rise in intra-abdominal pressure. Low PDF volume is used after the PD catheter placement to avoid leakage. The volume is gradually increased over the next three or four days as tolerated by the patient.

Inflow time is the time required to instill the PD fluid into the peritoneal cavity under the effect of gravity. The time is usually 10-15 minutes [25]. It depends on amount of fluid to be infused, height of the bag from patient's abdomen, and resistance to flow due to the kinking of the catheter or reduced bowel motility. The inflow time should be kept to minimal to maximize efficiency of peritoneal dialysis.

Dwell time is the time period for which the exchange volume stays in the intraperitoneal cavity for diffusion and ultrafiltration which is usually 30 minutes in single acute peritoneal dialysis exchange. A dwell time of less than 30 minutes is usually not adequate [35]. The dwell time for patients on acute CPD is about 3-6 hours which can be 
TABLE 6: Dialysis fluid glucose concentration.

\begin{tabular}{lcc}
\hline Glucose (monohydrate) & Fluid osmolarity & Ultrafiltrate volume \\
\hline $\mathrm{g} / \mathrm{dL}$ & $\mathrm{mOsm} / \mathrm{L}$ & mL per exchange over one hour \\
$1.5 \mathrm{~g} / \mathrm{dL}$ & 346 & $50-150$ \\
$2.5 \mathrm{~g} / \mathrm{dL}$ & 396 & $100-300$ \\
$4.25 \mathrm{~g} / \mathrm{dL}$ & 485 & $300-400$ \\
\hline
\end{tabular}

shortened to increase the total number of exchanges to improve solute clearance.

Outflow time is the time required to drain effluent dialysate after dwell which takes place under effect of gravity. It is usually takes 20-30 minutes to complete [25]. Outflow is affected by volume of the effluent to be drained, outflow resistance to drainage, and height difference between patient and drainage bag. One should ensure complete drainage as incomplete drainage can cause a rise in intra-abdominal pressure causing respiratory embarrassment or abdominal discomfort.

The number of exchanges depends on the amount of fluid and solute removal required for a particular patient. The usual number of exchanges is about 24 per day with standard acute PD and approximately 4-6 per day with CPD.

Some drugs can be added to the PD fluid to treat certain specific conditions. Some of these drugs are the following.

Heparin. Heparin is used to prevent clot formation. Usually a dose of 500 units/liter is used [36]. Usually, heparin is also used when plugs or strands of fibrin are visible on the drained fluid. Heparin is more beneficial when added prophylactically. No systemic anticoagulation risk exists when heparin is used through a PD catheter as there is no systemic absorption of heparin through peritoneum.

Insulin. Usually insulin is used in diabetic patients on $\mathrm{PD}$ for glycemic control. The glucose content of the PD fluid can worsen hyperglycemia especially with hypertonic fluid, which can result in very high blood glucose levels. Intraperitoneal insulin is usually added to the PD fluid, and the dose is adjusted based on frequent blood glucose monitoring. Insulin should not be added in last 2-3 exchanges to prevent postdialysis hypoglycemia.

A usual regimen comprises of an increasing insulin dose in the dialysis bag with increasing glucose concentration as follow:

4-5 units/L for $1.5 \mathrm{~g} / \mathrm{dL}$ PD fluid,

5-7 units/L for $2.5 \mathrm{~g} / \mathrm{dL}$ PD fluid,

7-10 units/L for $4.25 \mathrm{~g} / \mathrm{dL}$ PD fluid.

Potassium. Normally, there is no Potassium in the dialysis fluid but potassium can be added to the PD fluid in hypokalemic patients. Usually $3-4 \mathrm{meq} / \mathrm{L}$ is added to maintain normokalemia [36].

Antibiotics. Intraperitoneal administration of antibiotics is efficient and provides an alternative route for patients with poor vascular access and for those with peritonitis. A variety of antibiotics can be administered through PD intraperitoneally. It is very important to maintain accurate flow sheets, maintain intake/output charts, and document net ultrafiltration in patients on acute PD. Intraperitoneal route is preferred to intravenous dosing for treating peritonitis. Both intermittent and dosing of antibiotics are equally efficacious. Empiric treatment of peritonitis should start immediately and should have both gram-positive and gramnegative coverage. In case of intermittent therapy, these antibiotics should be given intraperitoneally and allowed to dwell for 6 hours. First generation cephalosporin or Vancomycin can be used for gram-positive coverage and either third generation cephalosporin or aminoglycoside should be used to cover gram-negative organisms. Results of culture and sensitivity should be followed and antibiotics should be changed based on sensitivity of the organism. Most patients show considerable clinical improvement within 48 hours of initiation of antibiotic treatment. Total duration of antibiotic therapy for peritonitis depends upon the organism isolated and ongoing results of peritoneal fluid cell count and repeat culture. The reader should refer to International Society of Peritoneal Dialysis guidelines regarding doses of various antibiotics used intraperitoneally in $\mathrm{PD}$ for treatment of peritonitis [37].

\section{Complications of PD}

Acute PD may be associated with infectious, mechanical, or medical complications of varying severity $[38,39]$. A brief overview of the complications is discussed in this section.

7.1. Infectious Complications. Peritonitis occurs in up to $12 \%$ of cases frequently developing within first 48 hours of therapy [40]. It usually occurs with an open drainage system and is due to contamination of the connection or disconnection of each new exchange. It can be caused by both gram-positive and gram-negative bacteria. Peritonitis is usually suspected when the effluent is cloudy. The diagnosis is confirmed by PDF analysis for cell count, gram staining, and culture and sensitivity. Antibiotic therapy should be initiated as soon as possible empirically to avoid serious consequences of peritonitis like sepsis, catheter removal, and so forth. One can narrow down the antibiotic administration based on results of culture and sensitivity of PDF.

\subsection{Mechanical Complications}

Pain. Pain is usually experienced at the incision site or may be associated with manipulation of the catheter during the 
TABLE 7: Acute peritoneal dialysis orders.

Nephrologist should make sure that PD catheter is adequately inserted preferably chronic catheter and has no issues with flow of the fluid. PD orders need to be individualized depending upon hemodynamic status of the patient, laboratory work, and volume status. PD orders need to be reviewed and written daily as patients with AKI usually fluctuate acid base and electrolyte balance daily

Nursing orders

Dialysis session length ...... hours

Dialysis volume per exchange ...... L

Dialysis dextrose concentration \%

Inflow time ..... min Dwell time ..... min, Outflow time ..... min

Vital signs q..... hours

Weigh patient q..... hours

Warm dialysate fluid to body temperature

Maintain strict intake and output

Additives to dialysate Heparin yes/no, Insulin yes/no, Potassium yes/no

Medication dose frequency

Vancomycin ..... mg/L of exchange, Tobramycin ..... mg/L of exchange other antibiotic ..... mg/L

Catheter care and dressing change every day

Full chemistry panel including blood glucose level to be done every 12 hours each day during dialyisis

Send $15 \mathrm{cc}$ of dialysate fluid from catheter every morning during dialysis and send it for cell count with differential, gram staining, and culture and sensitivity yes/no

Renal Physician to be notified immediately for the following situations:

Poor dialysate flow

Severe abdominal pain or distention

Change in color of dialysate, bloody, or cloudy drainage

Dialysate leak or purulent drainage around catheter exit site

Patient hypotensive with systolic blood pressure of $<\ldots . . \mathrm{mm} \mathrm{Hg}$

Respiratory rate of $\geq \ldots$. per minute or severe shortness of breath in non ventilated patient

Temperature of $\geq \ldots$. C

Two consecutive positive exchanges

Single positive exchange balance (dialysate IN-dialysate OUT) of $>1000 \mathrm{~mL}$

If negative balance exceeds ..... L L over ...... hours

Notification of abnormal laboratory values

procedure. Pain may be due to multiple factors such as low $\mathrm{pH}$ of PD fluid, low temperature, the jet flow from a straight catheter tip, or distension of the tissue around the catheter. This pain may be minimized by infusion of alkaline PD fluid with the addition of sodium bicarbonate and by raising the temperature of the PDF by warming or slowing down the rate of the infusion of fluid.

Perforation. Perforation of various abdominal viscera can occur during the insertion of the peritoneal dialysis catheter. Usual organs at risk of perforation are bowel and urinary bladder. Bowel perforation is manifested by severe abdominal pain, leak of intestinal contents, or urine in case of urinary bladder perforation through the catheter stylet. The diagnosis of the perforated organ may be evident immediately after the event or it may remain silent for some time, leading to other complications. Peritoneoscopic or surgical implantation of the catheter should reduce the incidence of perforation.
Blood in Dialysate. Bloody dialysate can occur due to a bleeding tendency in the patient's body. Bleeding can result from laceration of anterior abdominal wall vessels (i.e., inferior hypogastric artery) or less frequently, puncture of intraabdominal vessels. The treatment of bleeding depends on its severity. Usually, frequent exchanges and use of intraperitoneal heparin to prevent clotting are generally used until the effluent clears or surgical intervention is deemed necessary. Bleeding due to laceration of the abdominal wall can be controlled through ligation of the vessel through either laparoscopy or laparotomy.

Early Dialysate Leakage. Incidence of pericatheter leaks can vary from $0-40 \%$. Pericatheter leaks may not be apparent in the immediate postinsertion period unless a full exchange is performed. Catheter leak usually occurs due to the presence of certain predisposing factors like old age, obesity, diabetes mellitus, chronic steroid use, and multiparity. It is usually avoided by using low fill volumes. The risk of leak is low in 
supine position because intraperitoneal pressure is the lowest in this position as compared to sitting or standing. Catheter leaks can be prevented with use of tightly secured purse string sutures at the site of entrance of the catheter into the peritoneal cavity and by precisely placing the catheter cuffs. Sutures should never be placed at exit site skin to prevent leak.

Respiratory Insufficiency. Respiratory distress usually occurs due to abdominal distension as the instillation of peritoneal fluid causes a rise in intra-abdominal pressure. It can be prevented by careful observation to ensure complete emptying during the allowed drainage period.

Extravasation of Fluid in Tissue Compartments. Extravasation of fluid is usually in the abdominal wall. This sometimes presents as genital edema. It is usually due to peritoneal defects at the site of catheter insertion. It is suspected when there is a reduced amount of drainage volume, increased abdominal girth, and increased body weight without edema appreciated elsewhere in the body. Imaging studies are needed to diagnose the underlying cause of the extravasation.

Hydrothorax. PD-related Hydrothorax was first described in 1967 by Edwards and Unger [41]. The prevalence of Hydrothorax varies ranging from $1.6 \%$ [42] to $6 \%$ of adult PD patients [43]. Usually pleural effusion is transudate in nature, and it occurs usually immediately after starting PD or a few days later [44]. Hydrothorax is usually right sided and is due to the presence of a congenital diaphragmatic defect with a subsequent pleuroperitoneal communication, disorder of lymphatic drainage, and pleuroperitoneal pressure gradient [44-46]. The glucose concentration of the pleural fluid is usually greater than the glucose level in the serum although this is not always a consistent finding. Diagnosis of pleuroperitoneal communication may require use of radionuclide scanning with technetium. Severe cases may require cessation of $\mathrm{PD}$ and surgical closure of the communication, pleurodesis by talc insufflation, or videoassisted thoracoscopic pleurodesis.

\subsection{Medical Complications}

Fluid, Electrolyte, and Acid Base Disturbances. Complications related to fluid and electrolyte can be avoided by close monitoring of the changes in patient's body weight, documentation of dialysis regimen pertaining to dialysis exchange frequency, osmotic strength of fluid used, volume of PDF used per exchange, and ultrafiltration. Daily intake and nonurinary output records should be reviewed on a daily basis. Hypernatremia is usually caused by the sieving effect with ultrafiltrate being formed with much lower concentration of sodium than that of plasma. Hypernatremia usually can be alleviated by the use of glucose containing fluids. Lactic acidosis is rare except in patients with terminal liver failure. Lactic acidosis can be avoided by the use of bicarbonate containing peritoneal dialysis fluid in patients with terminal liver failure. Occasionally, severe lactic acidosis can be due to ongoing bowel ischemia and should be excluded under relevant clinical conditions.

Hypervolemia and Hypovolemia. Volume changes can occur due to the use of either hyperosmotic fluid or due to ultrafiltration failure. This can be alleviated by adjusting the prescription of dialysis or in some situations may require temporary cessation of dialysis. Hypervolemia due to ultrafiltration failure is usually seen in high solute transporters. This particular ultrafiltration failure can be seen during episodes of acute peritonitis.

Hypoalbuminemia. Albumin loss is seen due to high protein losses in the dialysate especially during episodes of peritonitis. These losses can be as high as 10-20 grams a day and patients may require protein supplementation either orally or intravenously.

\section{Outcome of Patients on Acute Peritoneal Dialysis}

This section will review recent studies conducted in AKI patients treated with acute PD alone for dialytic therapy and the studies comparing acute PD with other modalities of renal replacement therapies. Two recent studies conducted in AKI patients using acute PD have given promising results. One prospective study [47] with $30 \mathrm{AKI}$ patients was performed in Brazil. The purpose of the study was to explore role of high volume continuous peritoneal dialysis (CPD) in patients with AKI, analyzing metabolic abnormality, resolution of fluid balance, and patient outcome. All patients had AKI due to ischemic or nephrotoxic Acute Tubular Necrosis (ATN). Adequacy of dialysis was measured by formula $\mathrm{Kt} / \mathrm{V}$ (where $\mathrm{K}$ is rate of urea clearance by peritoneal membrane in litres, $t$ is the treatment duration (24 hours), and $\mathrm{V}$ is volume of body urea distribution in litres). The prescribed $\mathrm{Kt} / \mathrm{V}$ value was 0.65 per session. In this study patients were assigned to high-dose continuous peritoneal dialysis (CPD) via a flexible PD catheter and automated $\mathrm{PD}$ with a cycler. PD exchanges were done with two liter peritoneal fluid using 35-50 minute dwell time with total of 36-44 Liters of PDF per day. Biochemical parameters (Urea, Creatinine, $\mathrm{pH}$, Potassium and Bicarbonate) were measured twice daily. Patients received $236 \mathrm{CPD}$ sessions resulting in normalized creatinine clearance and urea $\mathrm{Kt} / \mathrm{V}$ values of $110 \pm 22.5$ and $3.8 \pm 0.61$ per week per $1.73 \mathrm{~m}^{2}$ body surface area, respectively. After 4 days of $\mathrm{CPD}$, patients had stable BUN value $<50 \mathrm{mg} / \mathrm{dL}$ and creatinine at $<4 \mathrm{mg} / \mathrm{dL}$. In this study, $57 \%$ of the patients died and $23 \%$ of the patients recovered their renal function. The authors concluded that high-dose CPD using flexible PD catheter and automated PD with a cycler was an effective therapy for AKI which provides appropriate metabolic and acid-base control as well as adequate dialysis dose and fluid removal.

Another prospective, randomized, crossover study [48] from India enrolled 87 hemodynamically stable patients with AKI ( $88 \%$ of AKI in this study was due to medical reasons). Two different modalities of PD, Tidal PD (TPD), 
versus continuous peritoneal dialysis (CPD) were done in patients with mild to moderate hypercatabolism. Severely hypercatabolic and hemodynamically unstable patients were excluded from the study. Patients received either TPD or CPD after insertion of rigid peritoneal dialysis catheter. If there was a need to continue PD in patients with no recovery of renal function, patients were crossed over to other modality of PD after washout period of 14 hours. Total volume of PDF used daily was $26 \mathrm{~L}$ by using $2 \mathrm{~L}$ bags with dwell time of 210 minutes in CPD group and 10-minute dwell in TPD group. Patients completing at least one set of dialysis (CEPD + TPD or TPD + CPD) were included in final analysis. The study showed that different modalities of PD are adequate methods to maintain BUN levels at $65 \mathrm{mg} / \mathrm{dL}$ in mild to moderate hypercatabolic AKI patients from developing countries. Other studies done in the past in patients with AKI have shown similar results [24, 49].

Studies comparing acute PD to other modalities of renal replacement therapy are limited and the results of the conducted studies are conflicting. Phu et al. [50] performed an open, randomized comparison of pumped venovenous hemofiltration and peritoneal dialysis in patients with infection-associated AKI in an infectious disease referral hospital in Vietnam. The primary outcome in this study was to assess rapidity of resolution of metabolic abnormalities like correction of academia and creatinine level between the two modalities in patients with AKI. Secondary end points were death, need for further renal replacement therapy, incidence of serious complications, and cost of treatment. This study included patients with AKI due to sepsis (48 patients with severe Falciparum Malaria, 22 patients with sepsis). Children under age of 15 years, pregnant females, and patients who received renal replacement therapy previously were excluded from the study. This trial recruited 70 patients over a period of five years (1993-1998). PD was performed by use of rigid peritoneal catheter done under local anesthesia, and open drainage system was used with dwell time of 30 minutes. Daily PD fluid volume of $70 \mathrm{~L}$ was used. Hemofiltration was performed through insertion of femoral catheter at blood flow rate of $150 \mathrm{cc} / \mathrm{min}$, and CVVH effluent rate was approximately $25 \mathrm{~L} /$ day. Renal replacement therapy was continued till attending physician decided that it was no longer indicated. Duration of Hemofiltration was half as compared to duration of peritoneal dialysis. Results of the study showed that rate of correction of metabolic abnormalities was twice as fast in Hemofiltration group as compared to patients on peritoneal dialysis. Mortality rate of patients (one of the secondary end points in the study) on Peritoneal Dialysis was $47 \%$ compared to $15 \%$ on CRRT. The need for further renal replacement therapy (another secondary end point) was higher in survivors of PD than those of Hemofiltration. This trial had a small number of patients in shock (3\% versus $5 \%$ in $\mathrm{PD}$ and Hemofiltration group, resp.). However, use of intermittent $\mathrm{PD}$ with rigid catheters, an open drainage system, and manual exchanges may have led to inadequate solute clearance and hence high mortality rate in the PD group. Interestingly, the same authors had published another study in 1992 showing a significant reduction in mortality of AKI patients treated with acute PD associated with malaria. In this study, PD was the only modality used as there was no other dialytic modality available in this tertiary care [51].

Another prospective, randomized, controlled trial from Brazil compared high volume Peritoneal Dialysis (HVPD) with daily Hemodialysis (DHD) in RRT of AKI due to ischemic ATN associated with sepsis in the majority of the patients $[52,53]$. The primary outcome in this study was to compare patient outcome for mortality rate and recovery of renal function. Secondary end point was to examine the adequacy of HVPD and DHD in relation to metabolic control. In this study, intermittent Hemodialysis was given 6 days a week with targets $\mathrm{Kt} / \mathrm{V}$ of 1.2 even though delivered dialysis dose was much lower in HVPD patient (weekly Kt/V = 3.6) as compared to DHD group (weekly $\mathrm{Kt} / \mathrm{V}=4.8$ ). Peritoneal dialysis was performed 24 hours a day 7 days per week by using a flexible peritoneal dialysis catheter with 30 55-minute dwell time assisted with use of automated cycler. The prescribed Kt/V value was 0.65 in CPD group and total of 36-44 L per day of PD fluid was used. DHD was performed daily with at least 3-hour session six times a week using double lumen temporary dialysis catheter. A total of 120 patients out of 154 completed the trial from 2004 to 2006 with 60 patients enrolled in each arm of the study. $70 \%$ of the enrolled patients had hemodynamic instability. Mean number of sessions was 5.5 in HVPD and 7.5 in DHD group. Hospital mortality was $58 \%$ in patients who were treated with high volume peritoneal dialysis and $53 \%$ in patients who were randomly assigned to daily hemodialysis $(P=.71)$. $83 \%$ of surviving patients in the peritoneal dialysis group recovered kidney function as compared with $77 \%$ in the hemodialysis group and time to renal recovery was shorter in HVPD group as compared to DHD (7.2 \pm 2.6 versus $10.6 \pm 4.7$ days, $P=.04)$. Rate of catheter-related infection was similar between the two groups. There was no significant difference in patient survival after 30 days of treatment (50\% survival rate in each group). This outcome is different from the study mentioned earlier.

There are no studies comparing the outcome of peritoneal dialysis as compared to alternate day intermittent hemodialysis in AKI. Hence, peritoneal dialysis remains an acceptable alternative to hemodialysis and CRRT especially in countries where technology for IHD and CRRT is not readily available.

\section{Conclusion}

Peritoneal dialysis has been in use since 1970 in patients with acute kidney injury especially those who are hemodynamically unstable or at risk of bleeding because of bleeding tendency, in pediatric patients with acute kidney injury, and in patients with vascular access failure.

Peritoneal dialysis remains an effective therapy which is simple and easy to use. This is especially the case for infants and children with AKI both in ICU and non-ICU settings, although its use is less preferable in western countries especially with advent of newer options available for CRRT like SLED and CVVDHF. It is a less effective modality 
in certain clinical situations like patients with poisoning, hypercatabolic states, and pulmonary edema. There is a limited data concerning the effect on mortality of PD versus other RRT therapies like intermittent hemodialysis and other continuous renal replacement therapies in patients with acute kidney injury.

\section{References}

[1] H. R. Brady and G. G. Singer, "Acute renal failure," The Lancet, vol. 346, no. 8989, pp. 1533-1540, 1995.

[2] M. H. Maxwell, R. E. Rockney, C. R. Kleeman, and M. R. Twiss, "Peritoneal dialysis. 1. Technique and applications," Journal of the American Medical Association, vol. 170, no. 8, pp. 917-924, 1959.

[3] K. D. Nolph, Z. J. Twardowski, R. P. Popovich, and J. Rubin, "Equilibration of peritoneal dialysis solutions during longdwell exchanges," Journal of Laboratory and Clinical Medicine, vol. 93, no. 2, pp. 246-256, 1979.

[4] W. Moncrief . W., K. D. Nolph, and J. R. P. Rubin Popovich, "Additional experience with continuous ambulatory peritoneal dialysis (CAPD)," Transactions of the American Society for Artificial Organs, vol. 24, pp. 476-483, 1978.

[5] V. M. Reznik, R. W. Griswold, B. M. Peterson, A. Rodarte, M. E. Ferris, and S. A. Mendoza, "Peritoneal dialysis for acute renal failure in children," Pediatric Nephrology, vol. 5, no. 6, pp. 715-717, 1991.

[6] K. D. Nolph, "Continuous versus intermittent therapy for acute renal failure. Peritoneal dialysis for acute renal failure," ASAIO Transactions, vol. 34, no. 1, pp. 54-55, 1988.

[7] C. M. Kjellstrand and K. Solez, "Treatment of acute renal failure," in Diseases of the Kidney, W. R. Schrier and W. C. Gottschalk, Eds., vol. 2, pp. 1385-1394, Little, Brown and Company, Boston, Mass, USA, 5th edition, 1993.

[8] R. D. Swartz, T. W. Valk, A. J. S. Brain, and C. H. Hsu, "Complications of hemodialysis and peritoneal dialysis in acute renal failure," ASAIO Journal, vol. 3, no. 3, pp. 98-101, 1980.

[9] R. Mactier, "Non-renal indications for peritoneal dialysis," Advances in peritoneal dialysis. Conference on Peritoneal Dialysis, vol. 8, pp. 141-144, 1992.

[10] G. Balldin and K. Ohlsson, "Demonstration of pancreatic protease-antiprotease complexes in the peritoneal fluid of patients with acute pancreatitis," Surgery, vol. 85, no. 4, pp. 451-456, 1979.

[11] A. Kauste, K. Hockerstedt, J. Ahonen, and H. Tervaskari, "Peritoneal lavage as a primary treatment in acute fulminant pancreatitis," Surgery Gynecology and Obstetrics, vol. 156, no. 4, pp. 458-463, 1983.

[12] A. D. Mayer, M. J. McMahon, and A. P. Corfield, "Controlled clinical trial of peritoneal lavage for the treatment of severe acute pancreatitis," New England Journal of Medicine, vol. 312, no. 7, pp. 399-404, 1985.

[13] J. B. Reuler and R. A. Parker, "Peritoneal dialysis in the management of hypothermia," Journal of the American Medical Association, vol. 240, no. 21, pp. 2289-2290, 1979.

[14] I. H. Khan, I. S. Henderson, and R. A. Mactier, "Hyperpyrexia due to meningococcal septicaemia treated with cold peritoneal lavage," Postgraduate Medical Journal, vol. 68, no. 796, pp. 129 131, 1992.

[15] B. Z. Horowitz, "The golden hour in heat stroke: use of iced peritoneal lavage," American Journal of Emergency Medicine, vol. 7, no. 6, pp. 616-619, 1989.
[16] J. E. Sánchez, T. Ortega, C. Rodríguez et al., "Efficacy of peritoneal ultrafiltration in the treatment of refractory congestive heart failure," Nephrology Dialysis Transplantation, vol. 25, no. 2, pp. 605-610, 2010.

[17] M. Nakayama, H. Nakano, and M. Nakayama, "Novel therapeutic option for refractory heart failure in elderly patients with chronic kidney disease by incremental peritoneal dialysis," Journal of Cardiology, vol. 55, no. 1, pp. 49-54, 2010.

[18] J. P. Ryckelynck, T. Lobbedez, B. Valette et al., "Peritoneal ultrafiltration and refractory congestive heart failure," Advances in Peritoneal Dialysis, vol. 13, pp. 93-97, 1997.

[19] B. G. Stegmayr, R. Banga, L. Lundberg, A. M. Wikdahl, and M. Plum-Wirell, "PD treatment for severe congestive heart failure," Peritoneal Dialysis International, vol. 16, supplement 1, pp. S231-S235, 1996.

[20] M. de Graaff, A. H. Zegwaard, M. M. Zweers et al., "The effects of a dialysis solution with a combination of glycerol/amino acids/dextrose on the peritoneal membrane in chronic renal failure," Peritoneal Dialysis International, vol. 30, no. 2, pp. 192-200, 2010.

[21] M. Asola, K. Virtanen, K. Någren et al., "Amino-acid-based peritoneal dialysis solution improves amino-acid transport into skeletal muscle," Kidney International. Supplement, no. 108, pp. S131-136, 2008.

[22] H. L. Tjiong, T. Rietveld, J. L. Wattimena et al., "Peritoneal dialysis with solutions containing amino acids plus glucose promotes protein synthesis during oral feeding," Clinical Journal of the American Society of Nephrology, vol. 2, no. 1, pp. 74-80, 2007.

[23] S. T. Brown, D. J. Ahearn, and K. D. Nolph, "Potassium removal with peritoneal dialysis," Kidney International. Supplement, vol. 4, no. 1, pp. 67-69, 1973.

[24] J. S. Cameron, C. Ogg, and J. R. Trounce, "Peritoneal dialysis in hypercatabolic acute renal failure," The Lancet, vol. 1, no. 7501, pp. 1188-1191, 1967.

[25] N. Lamiere, "Principles of peritoneal dialysis and its application in acute renal failure," in Critical Care Nephrology, C. Ronco and R. Bellomo, Eds., p. 1357, Kluwer Academic, Dodrecht, The Netherlands, 1998.

[26] A. H. Tzamaloukas, S. Garella, and J. A. Chazan, "Peritoneal dialysis for acute renal failure after major abdominal surgery," Archives of Surgery, vol. 106, no. 5, pp. 639-643, 1973.

[27] R. W. Steiner, "Continuous equilibration peritoneal dialysis in acute renal failure," Peritoneal Dialysis International, vol. 9, no. 1, pp. 5-7, 1989.

[28] P. H. Juergensen, A. L. Murphy, K. A. Pherson, W. S. Chorney, A. S. Kliger, and F. O. Finkelstein, "Tidal peritoneal dialysis to achieve comfort in chronic peritoneal dialysis patients," Advances in peritoneal dialysis. Conference on Peritoneal Dialysis, vol. 15, pp. 125-126, 1999.

[29] B. Piraino, F. Bender, and J. Bernardini, "A comparison of clearences on tidal peritoneal dialysis and intermittent peritoneal dialysis," Peritoneal Dialysis International, vol. 14, no. 2, pp. 145-148, 1994.

[30] E. V. Balaskas, S. Izatt, M. Chu, and D. G. Oreopoulos, “Tidal volume peritoneal dialysis versus intermittent peritoneal dialysis," Advances in Peritoneal Dialysis, vol. 9, pp. 105-109, 1993.

[31] A. Vychytil, T. Lilaj, B. Schneider, W. H. Horl, and M. HaagWeber, "Tidal peritoneal dialysis for home-treated patients: should it be preferred?" American Journal of Kidney Diseases, vol. 33, no. 2, pp. 334-343, 1999. 
[32] S. N. Wong and D. F. Geary, "Comparison of temporary and permanent catheters for acute peritoneal dialysis," Archives of Disease in Childhood, vol. 63, no. 7, pp. 827-831, 1988.

[33] N. O. Kronfol, "Acute peritoneal dialysis prescription," in Handbook of Dialysis, J. T. Daugirdas and T. S. Ing, Eds., p. 301, Little, Brown and Company, Boston, Mass, USA, 2nd edition, 1994.

[34] A. Dejardin, A. Robert, and E. Goffin, "Intraperitoneal pressure in PD patients: relationship to intraperitoneal volume, body size and PD-related complications," Nephrology Dialysis Transplantation, vol. 22, no. 5, pp. 1437-1444, 2007.

[35] J. Rubin, C. Adair, T. Barnes, and J. Bower, "Dialysate flow rate and peritoneal clearance," American Journal of Kidney Diseases, vol. 4, no. 3, pp. 260-267, 1984.

[36] S. Korbet, "Acute peritoneal dialysis prescription," in Handbook of Dialysis, P. B. Daugirdas and T. S. Ing, Eds., p. 382, Lippincott Williams and Wilkins, Baltimore, Md, USA, 4th edition, 2007.

[37] P. K.-T. Li, C. C. Szeto, B. Piraino et al., "Peritoneal dialysisrelated infections recommendations: 2010 update," Peritoneal Dialysis International, vol. 30, no. 4, pp. 393-423, 2010.

[38] C. A. Vaamonde, U. F. Michael, R. A. Metzger, and K. E. Carroll, "Complications of acute peritoneal dialysis," Journal of Chronic Diseases, vol. 28, no. 11-12, pp. 637-659, 1975.

[39] M. H. Gault, E. L. Ferguson, J. S. Sidhu, and R. P. Corbin, "Fluid and electrolyte complications of peritoneal dialysis. Choice of dialysis solutions," Annals of Internal Medicine, vol. 75 , no. 2, pp. 253-262, 1971.

[40] M. S. Korbet and O. N. Kronfol, "Acute peritoneal dialysis prescription," in Handbook of Dialysis, T. J. Daugirdas, G. R. Blake, and S. T. Ing, Eds., pp. 333-342, Lippincott Williams \& Wilkins, Philadelphia, Pa, USA, 3rd edition, 2001.

[41] S. R. Edwards and A. M. Unger, "Acute hydrothorax-a new complication of peritoneal dialysis," Journal of the American Medical Association, vol. 199, no. 11, pp. 853-855, 1967.

[42] Y. Nomoto, T. Suga, K. Nakajima et al., "Acute hydrothorax in continuous ambulatory peritoneal dialysis-a collaborative study of 161 centers," American Journal of Nephrology, vol. 9, no. 5, pp. 363-367, 1989.

[43] C. M. A. Van Dijk, S. G. Ledesma, and I. Teitelbaum, "Patient characteristics associated with defects of the peritoneal cavity boundary," Peritoneal Dialysis International, vol. 25, no. 4, pp. 367-373, 2005.

[44] R. G. Ramon and A. M. Carrasco, "Hydrothorax in peritoneal dialysis," Peritoneal Dialysis International, vol. 18, no. 1, pp. 540-546, 1998.

[45] A. P. C. Yim, T. W. Lee, I. Y. P. Wan, and C. Ng, "Pleuroperitoneal fistula," Annals of Thoracic Surgery, vol. 73, no. 4, p. 1327, 2002.

[46] P. Saillen, F. Mosimann, and J. P. Wauters, "Hydrothorax and end-stage chronic renal failure," Chest, vol. 99, no. 4, pp. 1010 1011, 1991.

[47] D. Ponce Gabriel, G. V. Ribeiro do Nascimento, J. T. Caramori, L. C. Martim, P. Barretti, and A. L. Balbi, "High volume peritoneal dialysis for acute renal failure," Peritoneal Dialysis International, vol. 27, no. 3, pp. 277-282, 2007.

[48] V. C. Chitalia, A. Fernandes Almeida, H. Rai et al., "Is peritoneal dialysis adequate for hypercatabolic acute renal failure in developing countries?" Kidney International. Supplement, vol. 61, no. 2, pp. 747-757, 2002.

[49] A. Katirtzoglou, P. Kontesis, and D. Myopoulou Symvoulidis, "Continuous equilibration peritoneal dialysis (CEPD) in hypercatabolic renal failure," Peritoneal Dialysis Bulletin, vol. 3, no. 4, pp. 178-180, 1983.
[50] N. H. Phu, T. T. Hien, N. T. H. Mai et al., "Hemofiltration and peritoneal dialysis in infection-associated acute renal failure in Vietnam," The New England Journal of Medicine, vol. 347, no. 12, pp. 895-902, 2002.

[51] T. T. M. Trang, N. H. Phu, H. Vinh et al., "Acute renal failure in patients with severe falciparum malaria," Clinical Infectious Diseases, vol. 15, no. 5, pp. 874-880, 1992.

[52] D. P. Gabriel, J. T. Caramori, L. C. Martim, P. Barretti, and A. L. Balbi, "High volume peritoneal dialysis vs daily hemodialysis: a randomized, controlled trial in patients with acute kidney injury," Kidney International. Supplement, no. 108, pp. S87S93, 2008.

[53] D. P. Gabriel, J. T. Caramori, L. C. Martim, P. Barretti, and A. L. Balbi, "High volume peritoneal dialysis vs daily hemodialysis: a randomized, controlled trial in patients with acute kidney injury," Kidney International. Supplement, vol. 29, supplement 2, pp. S62-S67, 2008. 


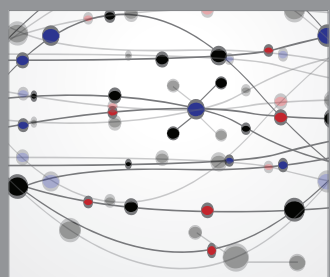

The Scientific World Journal
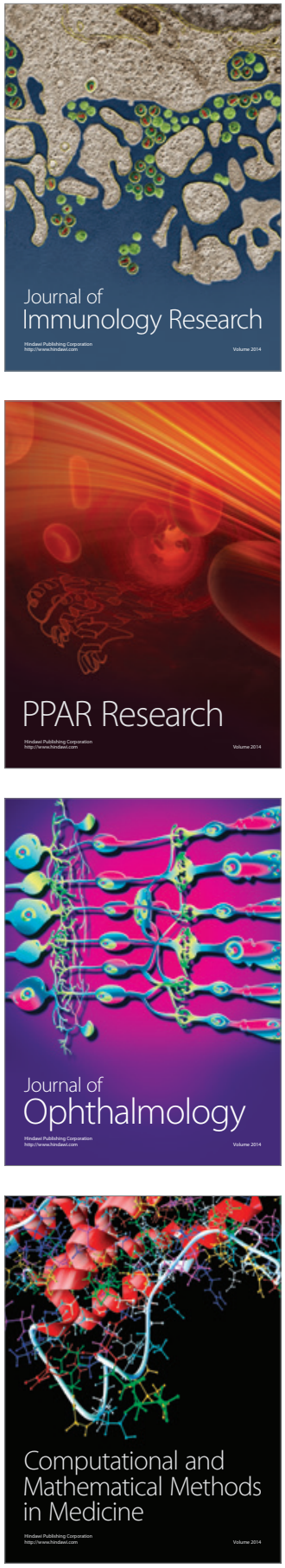

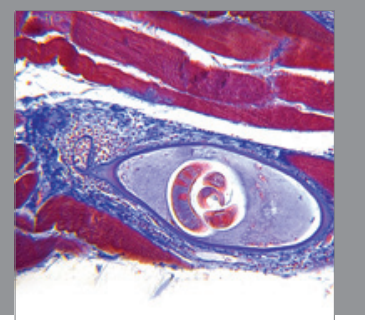

Gastroenterology

Research and Practice
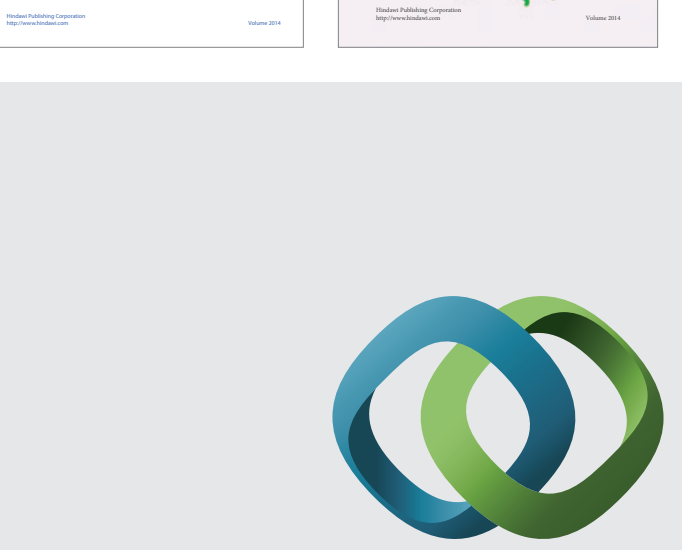

\section{Hindawi}

Submit your manuscripts at

http://www.hindawi.com
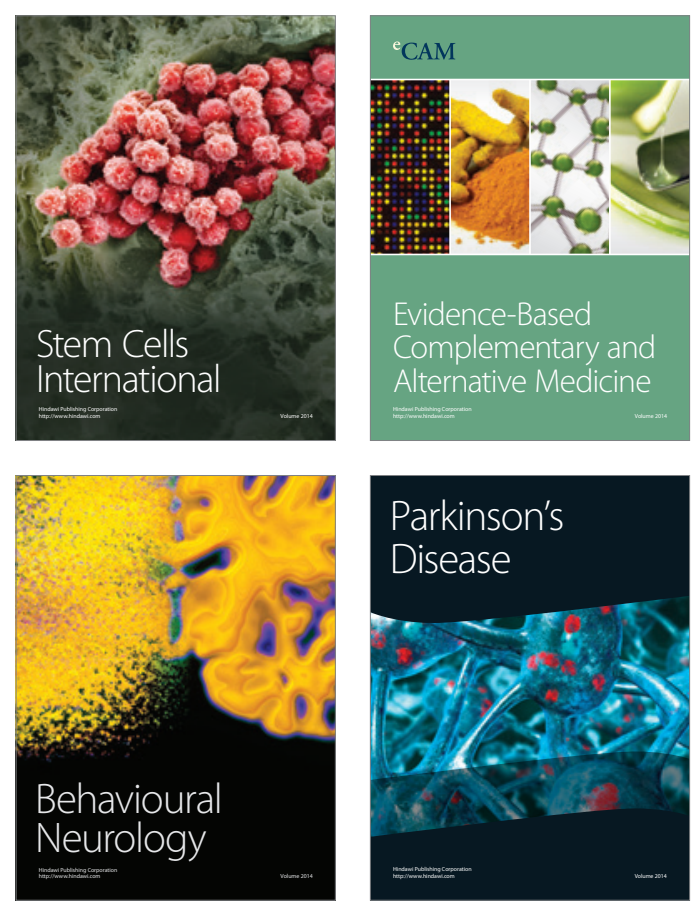

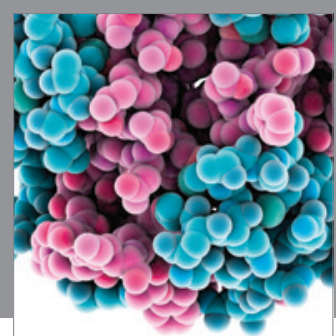

Journal of
Diabetes Research

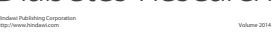

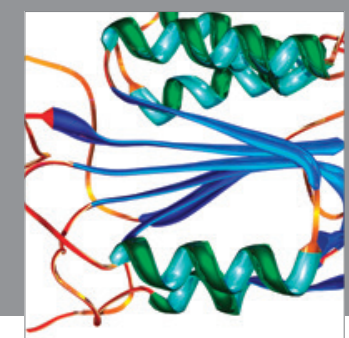

Disease Markers
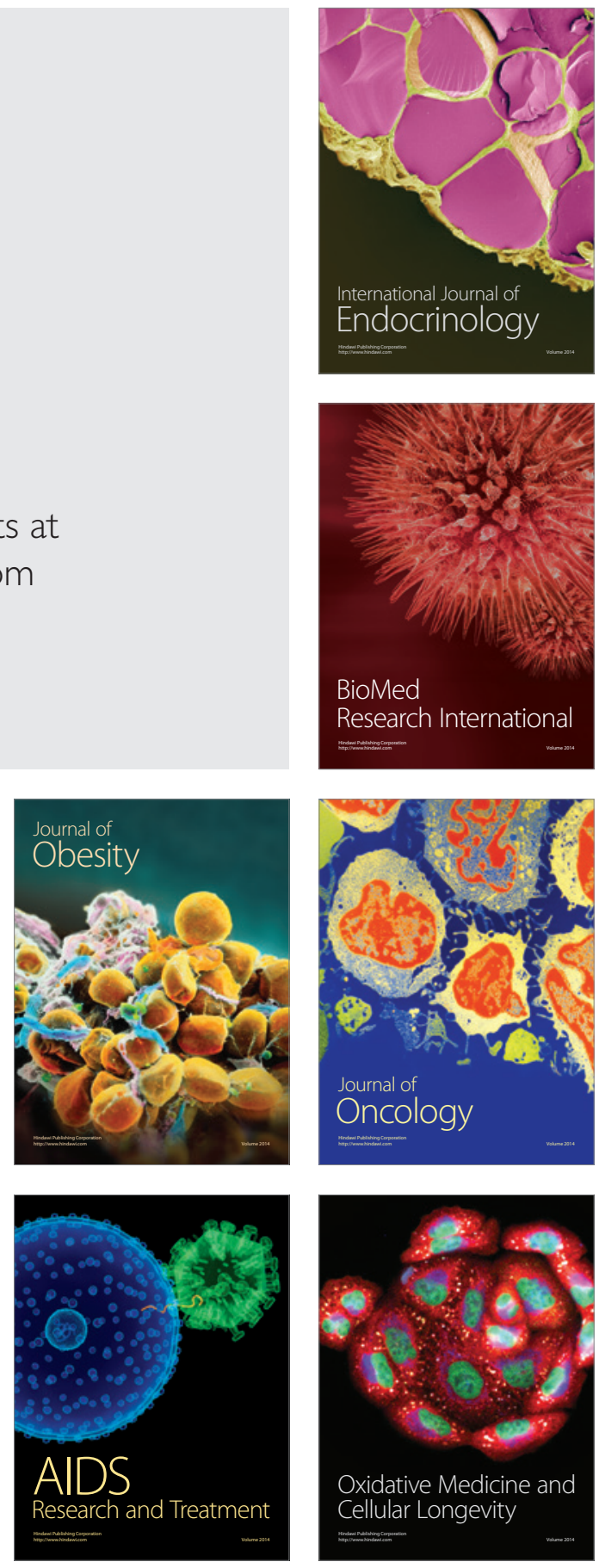